Method A retrospective analysis was performed on all abdominopelvic CT carried out in AMNCH, Tallaght from the 1st of January to 30st of June 2012. All patients with colonic BWT were included. Subsequently, Patients were excluded if they were under age of 18 years, history of Bowel Cancer and/or Prev colonic surgery, or if there was a specific CT finding related to the bowel other than BWT (mass, stricture, abscess, or confirmed cancer). Demographic information was obtained from $\mathrm{KEY}$ operating systems as well as $\mathrm{Hb}$ value, biopsy reports. Endoscopic reports were obtained from GI electronic reporting system as well as presence/absence of symptoms.

Results In total, 2,211 abdominopelvic CT reports were reviewed. After exclusion criteria were applied, 95 patients with colonic BWT were analysed. Of these, 52 had follow-on endoscopy. Demographic information revealed that 24 were male, with mean age of 56.8 years. The most common site of BWT reported was the sigmoid colon $(n=18)$, followed by descending colon $(n=10)$, and transverse colon $(n=7)$. Despite CT finding of BWT, 17 (32.6\%) had normal endoscopies. Inflammation/ Diverticulitis was found in $15(28.8 \%) / 10$ (19.2\%) endoscopies. $3(5.7 \%)$ were found to be adenomatous polyps. While, a further $8(15.3 \%)$ were colorectal cancer.

Conclusion Correlation between BWT on abdominopelvic CT and abnormal endoscopy at the same site was $67.4 \%$, in keeping with published studies.

\section{BOWEL WALL THICKENING ON CT; ITS SIGNIFICANCE AND RELATIONSHIP WITH ENDOSCOPIC ABNORMALITIES}

K Hartery, N Breslin Dept Gastroenterology, Adelaide Meath Hospital, Tallaght, Dublin 24, Ireland

\subsection{6/gutjnl-2013-305143.63}

Introduction Bowel wall thickening (BWT) is an increasing recognised entity seen on CT. Advances in technology and accumulated experience in image interpretation, even the most subtle changes affecting the bowel are now being detected.

Aims/Background "Further endoscopic evaluation" often is suggested when BWT is identified on CT imaging, Is there clinical correlation? 